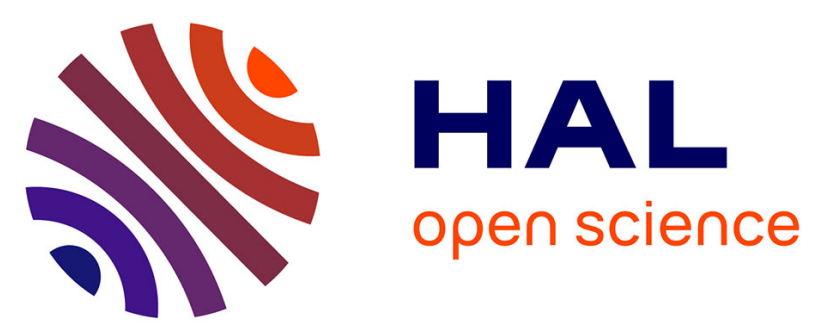

\title{
Synthesis of multivalent silica nanoparticles combining both enthalpic and entropic patchiness
}

\author{
Céline Hubert, Cyril Chomette, Anthony Désert, Ming Sun, Mona \\ Tréguer-Delapierre, Stéphane Mornet, Adeline Perro, Etienne Duguet, Serge \\ Ravaine
}

\section{To cite this version:}

Céline Hubert, Cyril Chomette, Anthony Désert, Ming Sun, Mona Tréguer-Delapierre, et al.. Synthesis of multivalent silica nanoparticles combining both enthalpic and entropic patchiness. Faraday Discussions, 2015, 181, pp.139-146. 10.1039/c4fd00241e . hal-01187264

\author{
HAL Id: hal-01187264 \\ https://hal.science/hal-01187264
}

Submitted on 25 Feb 2021

HAL is a multi-disciplinary open access archive for the deposit and dissemination of scientific research documents, whether they are published or not. The documents may come from teaching and research institutions in France or abroad, or from public or private research centers.
L'archive ouverte pluridisciplinaire $\mathbf{H A L}$, est destinée au dépôt et à la diffusion de documents scientifiques de niveau recherche, publiés ou non, émanant des établissements d'enseignement et de recherche français ou étrangers, des laboratoires publics ou privés. 


\title{
Synthesis of multivalent silica nanoparticles combining both enthalpic and entropic patchiness
}

\author{
Céline Hubert ${ }^{\mathrm{a}}$, Cyril Chomette ${ }^{\mathrm{b}}$, Anthony Désert ${ }^{\mathrm{b}, \mathrm{c}}$, Ming Sun ${ }^{\mathrm{b}, \mathrm{d}}$, Mona Treguer- \\ Delapierre $^{\mathrm{b}}$, Stéphane Mornet ${ }^{\mathrm{b}}$, Adeline Perro ${ }^{\mathrm{e}}$, Etienne Duguet ${ }^{\mathrm{b} *}$, Serge \\ Ravaine ${ }^{a *}$
}

\begin{abstract}
Silica particles with a controlled number of entropic patches, i.e. dimples, are synthesized through the growth of the silica core of binary multipods that have been produced by a seeded-growth emulsion polymerization reaction. Transmission electron microscopy studies indicate that the silica surface conforms to the shape of the polystyrene (PS) nodules of the multipods while growing, allowing a good control of the final shape of the dimpled silica particles. The PS nodules are also used as protecting masks to regioselectively graft amino groups, as revealed by the adsorption of gold markers. After dissolution of the PS nodules, some polymer chains remain grafted onto the silica surface, forming organic bumps. These residues are also selectively functionalized, leading to silica particles with both entropic and enthalpic patches.
\end{abstract}

\section{Introduction.}

Colloidal particles anisotropic in shape and/or in surface chemical functionality have attracted recent interest because of their potential use as building units for directed assembly into new materials. ${ }^{1-3}$ Among others, Glotzer et al. numerically studied the self-assembly of non-spherical and patchy colloids, i.e. particles with at least one portion of its surface area which differs in chemical functionality. ${ }^{4-6}$ In particular, they have predicted the formation of the diamond lattice, which could exhibit a full photonic band gap, ${ }^{7}$ according to the shape or the surface functionality of building blocks involved. They also distinguished the complementary concepts of entropic and enthalpic patches particles: the former describes topological surface irregularities capable to mechanically block directed interactions with other building blocks, e.g. corners, edges, dimples, etc. while the latter concerns "conventional" patches made of sticky chemical functions. ${ }^{6}$ Therefore, the selforganization of colloidal rods into nematic or smectic phases ${ }^{8,9}$ or of micron-sized cubic particles into colloidal crystals with a simple cubic symmetry ${ }^{10}$ may be seen as entropically controlled. Other recent examples concern the use of microparticles bearing several dimples capable of assembly with conventional spheres under depletion interactions. ${ }^{11-13}$

Several examples of enthalpically patchy particles have also been reported. Most of their synthesis routes are templating methods. ${ }^{14}$ Velegol et al. patterned particles with regions of predetermined size by the particle lithography technique. ${ }^{15}$ Kretzschmar et al. used the glancing angle deposition technique to create various patches in shape and/or in composition on polystyrene (PS) beads. ${ }^{16-18}$ This method was also used by Granick et al. to synthesize particles with two hydrophobic patches in order to self-assemble them in a kagome lattice. ${ }^{19}$
Starting from multipod-like silica/PS clusters ${ }^{20-22}$ and using the PS pods as temporary masks, ${ }^{23}$ we reported the synthesis of silica nanoparticles with 4, 6 and 12 dimples ideally arranged according to the repulsion figures of tetrahedron, octahedron and icosahedron, respectively. ${ }^{24} \mathrm{We}$ also shown that the silica surface between these entropic patches may be regioselectively functionalized.

Here, we extend this work to silica particles with 1, 2 and 3 dimples, because they are just as complementary building blocks for assembly into functional materials. We also report that we are able to selectively functionalize the organic residues located at the bottom of the dimples that remain after the dissolution of the PS nodules. Therefore, the as-obtained silica nanoparticles combine both enthalpic and entropic patchiness that can be tuned in order to either enhance particular desired particle alignment or produce competing forces. 


\section{Materials and methods}

Materials. Tetraethoxysilane (TEOS, $\geq 99 \%$, Fluka), N-[3(trimethoxysilyl)propyl]ethylenediamine (EDPS, 97\%, Aldrich), tetrakis(hydroxymethyl)phosphonium chloride (THPC, 80\%, Aldrich), n-propyltriethoxysilane (PTES, 97\%, Aldrich), paraformaldehyde (reagent grade, crystalline, Sigma-Aldrich), tin(IV) chloride (98\%, Aldrich), ethylene diamine $(99.5 \%$, Fluka), hydrochloric acid (37\%, Sigma-Aldrich) and ammonia (28-30\% in water, SDS) were used as received. Deionized water with a resistivity of $18.2 \mathrm{Ohm} . \mathrm{cm}$ at $25^{\circ} \mathrm{C}$ was obtained with a MilliQ system (Millipore). Tetrahydrofuran (THF), dimethylformamide (DMF), chloroform and absolute ethanol were purchased from Sigma-Aldrich.

Synthesis of dimpled silica particles. Multipods made of a central silica core and one, two, three, four, six or twelve PS satellite nodules, respectively, were prepared by seeded-growth emulsion polymerization of styrene, according to an already published procedure. ${ }^{20,21}$ The regrowth of the silica cores (step A) was performed in the following conditions: a certain volume

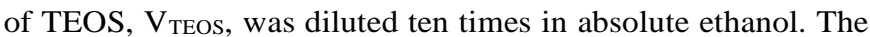
resulting solution was introduced continuously in a mixture made of $1 \mathrm{~mL}$ of an aqueous suspension of multipods, $45.5 \mathrm{~mL}$ of absolute ethanol and $3.2 \mathrm{~mL}$ of ammonia thanks to a singlesyringe pump $\left(0.5 \mathrm{~mL} \cdot \mathrm{h}^{-1}\right)$. The mixture was stirred at room temperature during $12 \mathrm{~h}$. The dissolution of the PS nodules (step B) was performed in $100 \mathrm{~mL}$ of $\mathrm{THF}$ at room temperature under stirring for $1 \mathrm{~h}$. After washing by three cycles of centrifugation/redispersion in THF, the multivalent silica particles were finally redispersed in absolute ethanol.

Functionalization of the surface area between the dimples. To graft amino groups onto the unprotected silica surface (step C), a given volume of EDPS corresponding to a nominal surface density of 20 functions.nm ${ }^{-2}$ was added directly into the particle suspension and the mixture was let to age at $40^{\circ} \mathrm{C}$ for 2 hours. The particle dispersion was purified by 3 cycles of centrifugation/redispersion in absolute ethanol. For the step C1, 5-nm gold nanoparticles, synthesized as previously described, ${ }^{25}$ were added into $20 \mathrm{~mL}$ of the functionalized multipod-like particles dispersion. The mixture was stirred at room temperature overnight, and then the particle assemblies were collected by centrifugation and washed three times with absolute ethanol. The dissolution of the PS nodules (step C2) was performed in $100 \mathrm{~mL}$ of THF at room temperature under stirring for 1 hour. After washing by three cycles of centrifugation/redispersion in THF, the multivalent silica particles were finally redispersed in absolute ethanol.

Functionalization of the PS residues at the bottom of the dimples. To graft propyl groups onto the unprotected silica surface (step D), a given volume of PTES corresponding to a nominal surface density of 20 functions.nm ${ }^{-2}$ was added dropwise into the particle suspension and the mixture was let to age at room temperature for $3 \mathrm{~h}$. The solvents were removed by using a rotavapor. An excess of THF was added to the solid residue to dissolve the PS nodules (step D1). After washing by three cycles of centrifugation/redispersion in THF, the silica particles were redispersed in chloroform. Finally, the particles were washed by three cycles of centrifugation/redispersion in 40 $\mathrm{mL}$ of chloroform. Step D2 consisted first in the chloromethylation of the PS chains by adding $5 \mathrm{~mL}$ of a $3 \mathrm{M}$ butyl chloromethylether solution in chloroform, prepared according to Warshawsky et al. ${ }^{26}$ and $0.3 \mathrm{~mL}$ of $\mathrm{SnCl}_{4}$ into $20 \mathrm{~mL}$ to the silica particles and the mixture was aged overnight at $45^{\circ} \mathrm{C}$. After washing by three cycles of centrifugation/redispersion in $\mathrm{HCl}$ solution (4 wt.\% in water) and three cycles of centrifugation/redispersion in a water/EtOH (50/50 wt \%), the silica particles were redispersed in $20 \mathrm{~mL}$ of DMF. Then, $1 \mathrm{~mL}$ of ethylenediamine was added and the mixture was aged overnight at $90^{\circ} \mathrm{C}$. After three cycles of centrifugation/redispersion in water, the $\mathrm{pH}$ was adjusted to 4 with $\mathrm{HCl}$. Finally, 5-nm gold nanoparticles were added into the suspension (step D3). The mixture was stirred at room temperature overnight, and then the particle assemblies were collected by centrifugation and washed three times with absolute ethanol.

Characterizations. TEM experiments were performed with a Hitachi H600 microscope operating at $75 \mathrm{kV}$. The samples were prepared as follows: colloidal dispersions were diluted in ethanol and one drop was deposited on a copper grid coated with a carbon membrane and left to evaporate.

\section{Results and Discussion}

Figure 1 exemplifies the multi-step approach developed to synthesize multivalent dimpled silica particles from PS/silica tetrapods. The number and position of the dimples depend directly on the number and position of the PS satellite nodules. The precursor PS/silica multipods are made of a silica core decorated by 1, 2, 3, 4, 6 or 12 PS nodules (Figure 3a-1, Table 1) and were synthesized by a seeded-growth emulsion polymerization of styrene. ${ }^{20-23}$ Their conversion into dimpled silica particles consists first in growing the silica core of the multipods used as endotemplates (step A). The PS nodules of the multipods were then dissolved in order to reveal the dimples (step B). The non-protected surface of the grown silica core can be selectively functionalized with amine groups (step C), as revealed by the regioselective absorption of gold nanoparticles (step C1), before the PS nodule dissolution (step C2). Because some PS are chemically grafted at the silica surface, they are present at the bottom of the dimples and can also be derivatized to improve their functionality. To do so, small alkyl chains have first to be grafted onto the non-protected silica surface (step D) before the PS nodules dissolution (step D1) to facilitate the dispersion of the silica particles in chloroform. The PS residues that remain in each dimple are then functionalized with chlorine and amine groups (step D2) through the chloromethylation of the phenyl groups followed by an amination reaction (Figure 2). Once again, the success of this regioselective functionalization may be revealed by the adsorption of gold nanoparticles (step D3). 


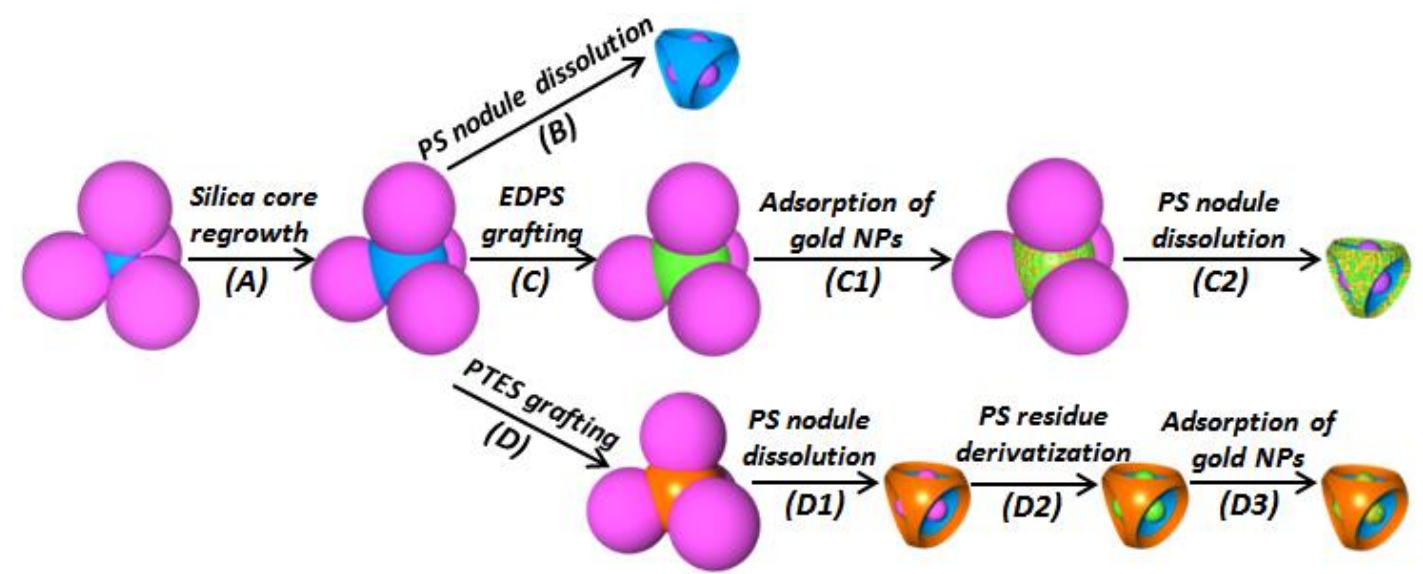

Fig. 1 Schematic representation of the multi-step approach for the synthesis and the regioselective functionalization of tetravalent silica particles.

$$
\left[\begin{array}{c}
\text { Chloro- } \\
\text { methylation }
\end{array}\right.
$$

Fig 2 Reactions of chloro-methylation and amination of the PS residues.

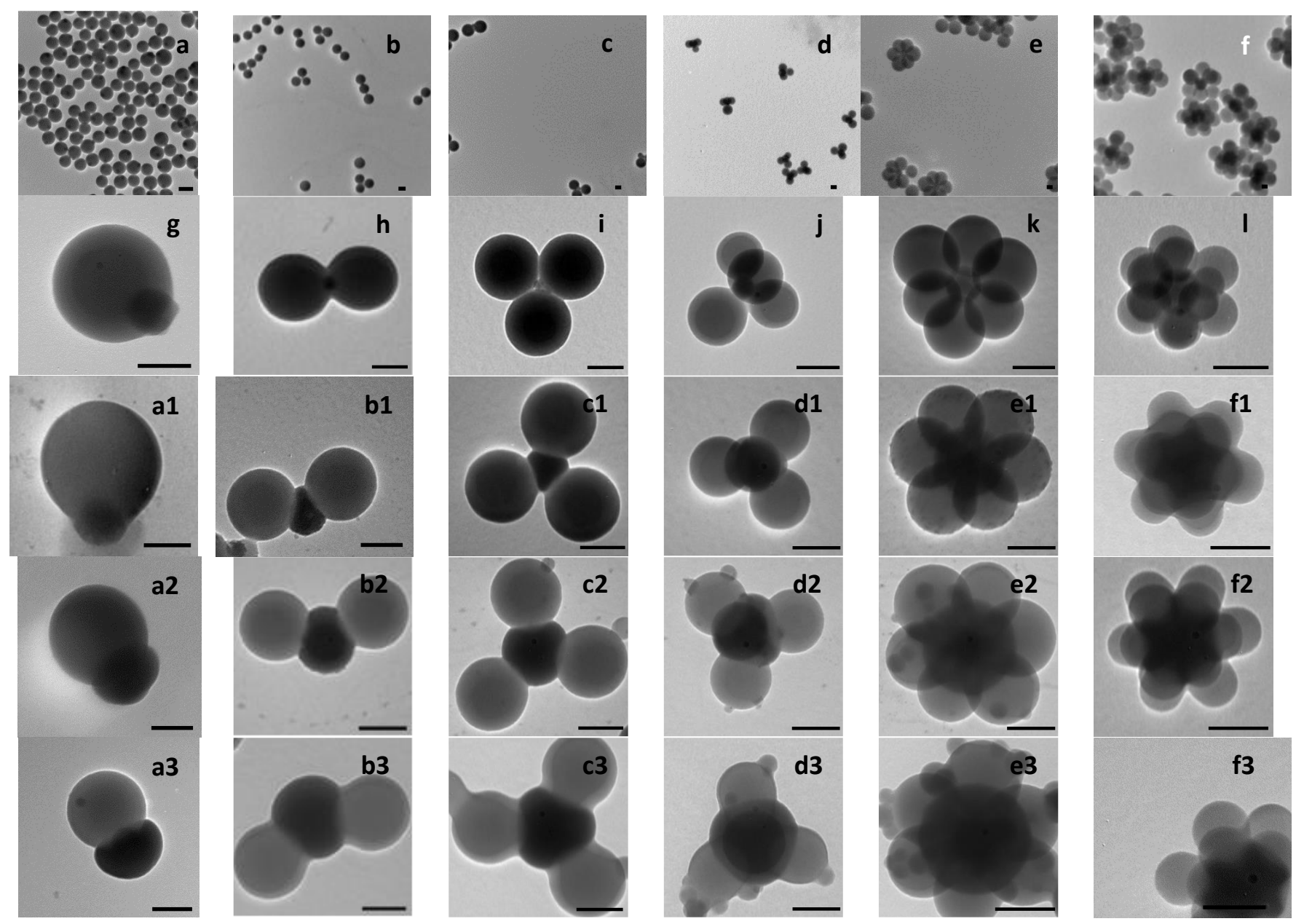

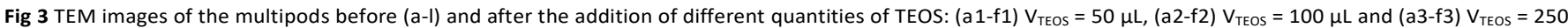
$\mu \mathrm{L}$. Scale bars: $100 \mathrm{~nm}$. 
The slow addition of a dilute solution of tetraethoxysilane in ethanol into a hydroalcoholic suspension of multipods, in presence of ammonia, led to the growth of the silica core conforming to the shape of the polystyrene nodules. As shown in Figure 3, the size of the silica cores can be tuned by adjusting the quantity of injected TEOS solution. Nevertheless, when this quantity is too high $(250 \mu \mathrm{L})$, the formation of silica protuberances at the surface of the polystyrene nodules is observed Figure $3 \mathrm{~d} 3$ and e3). The dependence of the size of the silica core on $\mathrm{V}_{\text {TEOS }}$ is reported in Table 2.

\begin{tabular}{cccc}
\hline Precursors & $\begin{array}{c}\text { Diameter of } \\
\text { silica core }(\mathbf{n m})\end{array}$ & $\begin{array}{c}\text { Diameter of } \\
\text { PS nodules }(\mathbf{n m})\end{array}$ & Morphology yield \\
\hline Monopods & 85 & 220 & $96 \%$ monopods, $4 \%$, bipods \\
Bipods & 41 & 182 & $68 \%$ bipods, $16 \%$ tripods, $16 \%$ monopods \\
Tripods & 43 & 157 & $66 \%$ tripods, 21 \% bipods, $13 \%$ monopods \\
Tetrapods & 54 & 129 & $84 \%$ tetrapods, 13\% tripods, 3 \% bipods \\
Hexapods & 85 & 136 & $83 \%$ hexapods, 13\% pentapods, $4 \%$ tetrapods \\
Dodecapods & 85 & 67 & $70 \%$ dodecapods, 17\% decapods, 13\% nonapods \\
\hline
\end{tabular}

Table 1 Characteristics of the multipod-like precursors

\begin{tabular}{|c|c|c|c|}
\hline \multirow{2}{*}{ Precursors } & $\mathrm{V}_{\text {TEOS }}=\mathbf{5 0} \boldsymbol{\mu L}$ & $\mathrm{V}_{\mathrm{TEOS}}=100 \mu \mathrm{L}$ & $\mathrm{V}_{\mathrm{TEOS}}=250 \mu \mathrm{L}$ \\
\hline & \multicolumn{3}{|c|}{ Average edge-length values of the silica core (nm) } \\
\hline Monopods ( $7.310^{12}$ part.) & 105 & 175 & 210 \\
\hline Bipods $\left(3.710^{13}\right.$ part. $)$ & 100 & 150 & 200 \\
\hline Tripods $\left(3.71^{13}\right.$ part. $)$ & 90 & 140 & 190 \\
\hline Tetrapods $\left(1.81^{13}\right.$ part. $)$ & 110 & 150 & 180 \\
\hline Hexapods ( $7.310^{12}$ part.) & 150 & 200 & 240 \\
\hline Dodecapods $\left(7.310^{12}\right.$ part. $)$ & 100 & 150 & 250 \\
\hline
\end{tabular}

Table 2 Average edge-length values of the silica core after the injection of various volumes of TEOS into the reactive medium containing the indicated number of multipods.

The subsequent dissolution of the PS nodules (Figure 1, step (B)) gives rise to silica particles with a fixed number of dimples (Figure 4). As these concave imprints are precisely localized around the particles, they can be used as entropic patches to endow the particles with directional interactions and predetermined "instructions" for assembly, making them similar to atoms which have valences. Morphology yields were equal to $94 \%, 67 \%, 66 \%, 82 \%, 84 \%$ and $69 \%$ for mono-, bi-, tri-, tetra-, hexa- and dodecavalent particles, respectively, as determined by statistical analysis of TEM images over at least 100 dimpled silica particles per batch.

We have already demonstrated that polystyrene nodules can be used as protecting masks to functionalize specifically the
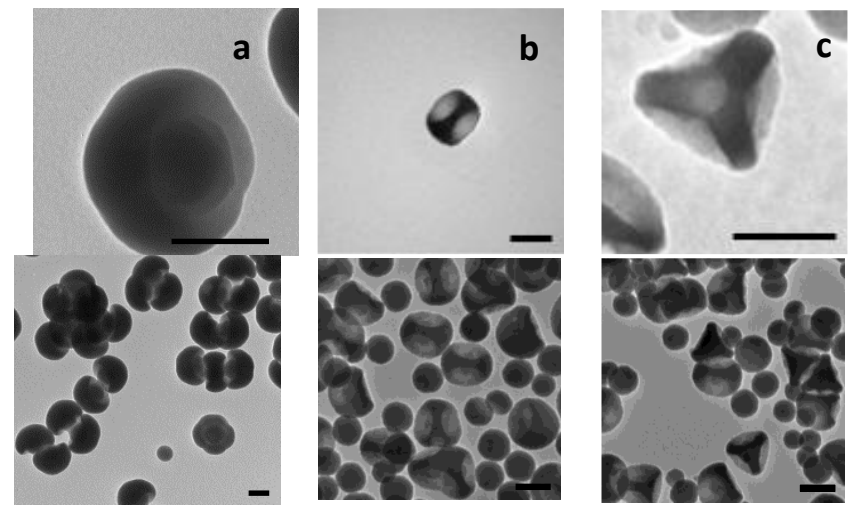

unprotected silica surface. ${ }^{23,24}$ According to this strategy, we functionalized the silica surface of the multipods after its growth with amino groups by adding EDPS at the end of the TEOS condensation (Figure 1, step (C)). The efficiency of this functionalization and of its regioselective character was evidenced by mixing the functionalized multipods with an aqueous suspension of THPC-stabilized 5-nm gold nanoparticles, ${ }^{25}$ which are known to adsorb specifically on amine groups (Figure 1, step (C1) and Figure 5 top). The last step of the synthesis was the dissolution of the polystyrene nodules revealing the presence of gold nanoparticles only on the unprotected silica surface (Figure 1, step (C2) and Figure 5 bottom).
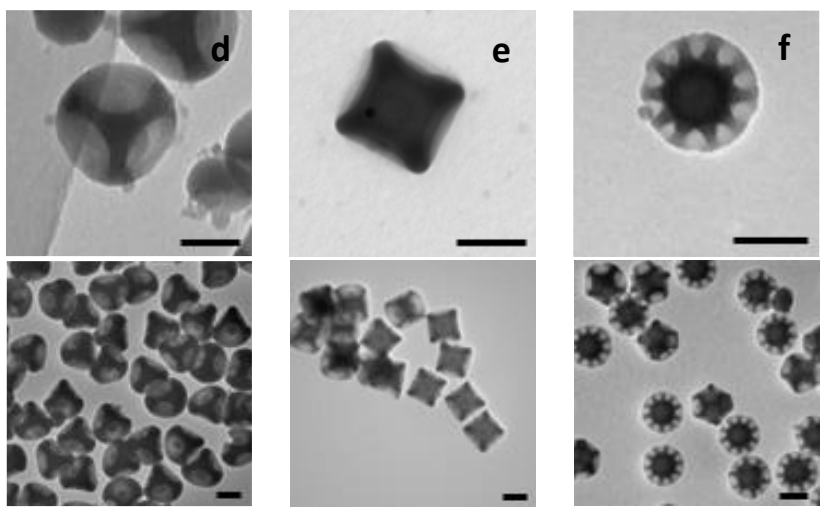

Fig 4 TEM images at different magnifications of silica particles with a) 1, b) 2, c) 3, d) 4, e) 6, f) 12 dimples. Scale bars: 100 nm. 


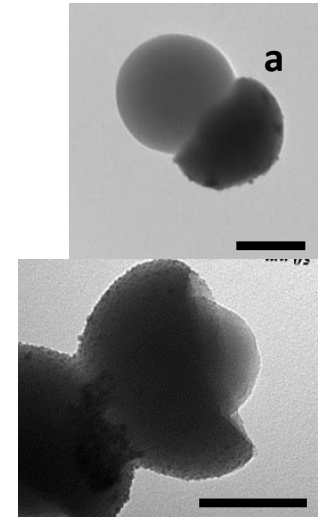

Fig 5 TEM images of the gold-c tripods, d) tetrapods, e) hexap

A closer look at Fig the bottom of each dimple after the PS nodules dissolution. The organic nature of these bumps was confirmed by using energy-dispersive X-ray spectroscopy (EDX) analyses coupled with scanning transmission electron microscopy (STEM) (Figure 6 a). We selectively modified these bumps through a chloromethylation reaction of their phenyl groups (Figure 1, step D2). The success of this functionalization step was first confirmed by EDX mapping (Figure 6 b) which shows the presence of chlorine atoms essentially at the bottom of the concave notches. We also grafted a diamine onto the chloromethylated macromolecules and revealed this regioselective functionalization by the specific adsorption of gold nanoparticles within the dimples (Figure 1, step (D3) and Figure 7 a-f).
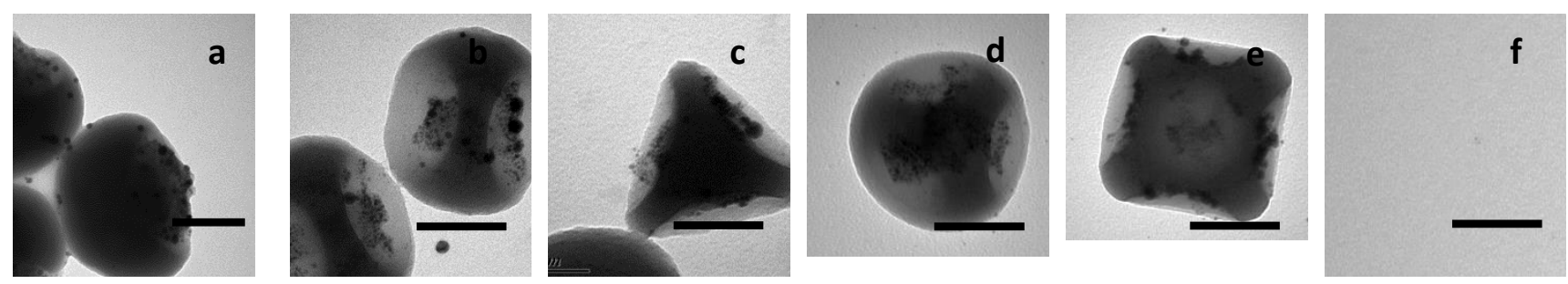

Fig 7 TEM images of silica particles with a) 1, b) 2, c) 3, d) 4, e) 6 and f) 12 gold-decorated bumps at the bottom of each dimple. Scale bars: 100 nm.

\section{Conclusions}

In summary, we generalized a method for preparing silica particles with a controlled number of dimples from 1 to 12 . We also showed that the size of these new dimpled silica particles can be easily controlled by adjusting the volume of the injected TEOS solution. The successful functionalization of both the nonprotected surface of the grown silica core and of the organic residues remaining at the bottom of each dimple was also demonstrated, thereby providing the possibility of getting particles with patches combining both enthalpic and entropic characters. These particles are promising building blocks for the fabrication of new lattices by self-assembly or as "lock" particles able to be combined with a precise number of "key" particles in each dimple in order to obtain new supracolloids. ${ }^{11-13}$

\section{Acknowledgements}

TEM experiments were performed at the Plateforme de Caractérisation des Matériaux and Bordeaux Imaging Center of the University of Bordeaux. This work was supported by the Conseil Régional d'Aquitaine, the Agence Nationale de la Recherche (grant ANR-07-BLAN-0271), and the LabEx AMADEus (ANR-10-LABX-42) in the framework of IdEx Bordeaux (ANR-10-IDEX-03-02), i.e. the Investissements d'Avenir programme of the French government managed by the Agence Nationale de la Recherche. 


\section{Notes and references}

${ }^{a}$ CNRS, Univ. Bordeaux, CRPP, UPR 8641, F-33600 Pessac, France.

${ }^{\mathrm{b}}$ CNRS, Univ. Bordeaux, ICMCB, UPR 9048, F-33600 Pessac, France.

${ }^{c}$ Univ. Bordeaux, CNRS, CBMN, UMR 5248, F-33600 Pessac, France.

${ }^{\mathrm{d}}$ School of Chemical Engineering and Light Industry, Guangdong

University of Technology, Guangzhou 510006, PR China.

${ }^{\mathrm{e}}$ Univ. Bordeaux, CNRS, ISM, UMR 5255, F-33405 Talence, France.

1 A. Van Blaaderen, Nature, 2006, 439, 545.

2 F. Li, D. P. Josephson and A. Stein, Angew. Chem. Int. Ed., 2011, 50, 360.

3 S. Sacanna and D. J. Pine, Curr. Opin. Colloid Interface Sci., 2011, 16, 96.

4 S. C. Glotzer, Science, 2004, 306, 419.

5 Z. Zhang and S. C. Glotzer, Nano Lett., 2004, 4, 1407.

6 G. van Anders, N. K. Ahmed, R. Smith, M. Engel and S. C. Glotzer, ACS Nano, 2014, 8, 931.

7 K. M. Ho, C. T. Chan and C. M. Soukoulis, Phys. Rev. Lett., 1990, 65, 3152.

8 H. Maeda and Y. Maeda, Phys. Rev. Lett., 2003, 90, 018303.

9 A. Kuijk, A. van Blaaderen and A. Imhof, J. Am. Chem. Soc., 2011, 133, 2346.

10 L. Rossi, S. Sacanna, W. T. M. Irvine, P. M. Chaikin, D. J. Pine and A. P. Philipse, Soft Matter, 2011, 7, 4139.

11 S. Sacanna, W. T. M. Irvine, P. M. Chaikin and D. J. Pine, Nature, 2010, 464, 575.

12 S. Sacanna, D. J. Pine and G.-R. Yi, Soft Matter, 2013, 9, 8096.

13 Y. Wang, Y. Wang, X. Zheng, G.-R. Yi, S. Sacanna, D. J. Pine and M. Weck, J. Am. Chem. Soc., 2014, 136, 6866.

14 A. B. Pawar and I. Kretzschmar, Macromol. Rapid Commun., 2010, 31, 150.

15 A. M. Yake, C. E. Snyder and D. Velegol, Langmuir, 2007, 23, 9069.

16 Z. He and I. Kretzschmar, Langmuir, 2013, 29, 15755.

17 A. B. Pawar and I. Kretzschmar, Langmuir, 2009, 25, 9057.

18 Z. He and I. Kretzschmar, Langmuir, 2012, 28, 9915.

19 Q. Chen, S. C. Bae and S. Granick, Nature, 2011, 469, 381.

20 A. Perro, E. Duguet, O. Lambert, J.-C. Taveau, E. Bourgeat-Lami and S. Ravaine, Angew. Chem. Int. Ed., 2009, 121, 367.

21 A. Désert, I. Chaduc, S. Fouilloux, J.-C. Taveau, O. Lambert, M. Lansalot, E. Bourgeat-Lami, A. Thill, O. Spalla, S. Ravaine and E. Duguet, Polym. Chem., 2012, 3, 1130.

22 A. Thill, A. Désert, S. Fouilloux, J.-C. Taveau, O. Lambert, M. Lansalot, E. Bourgeat-Lami, O. Spalla, L. Belloni, S. Ravaine, and E. Duguet., Langmuir, 2012, 28, 11575.

23 A. Perro, S. Reculusa, F. Pereira, M.-H. Delville, C. Mingotaud, E. Duguet, E. Bourgeat-Lami and S. Ravaine, Chem. Commun., 2005, 5542.

24 A. Désert, C. Hubert, Z. Fu, L. Moulet, J. Majimel, P. Barboteau, A. Thill, M. Lansalot, E. Bourgeat-Lami, E. Duguet and S. Ravaine, Angew. Chem. Int. Ed., 2013, 52, 11068.

25 D. G. Duff, A. Baiker and P. P. Edwards, Chem. Commun., 1993, 96.

26 A. Warshawsky and A. Deshe, J. Polym. Sci. Polym. Chem. Ed., 1985, 23, 1839 . 